\title{
Determination of Forces on Anchor Blocks and Stability Analysis in a 3 Dimensional Plane with Vector Approach
}

\author{
Gaurav Atreya ${ }^{a}$, Narendra Man Shakya ${ }^{b}$ \\ a Department of Civil Engineering, Pulchowk Campus, Institute of Engineering, Tribhuvan University, Nepal; \\ Currently at: Al Department, Eydean Inc., Kathmandu, Nepal \\ b Department of Civil Engineering, Pulchowk Campus, Institute of Engineering, Tribhuvan University, Nepal \\ Corresponding Email: a atreyagaurav98@gmail.com, b nms@ioe.edu.np
}

\begin{abstract}
The Anchor blocks are the structures that take most of the forces acting on the pipe bends and transfer it safely to the ground. Since the stability of the Anchor Blocks is a function of its weight, the economic design comes with accurate calculation of the forces. Although several sources have the design calculations for Anchor Blocks, there is room for improvements for 3D calculations. The calculation of the forces is hard to visualize in a two-dimensional plane as numerous planes have to be made for the analysis. The purpose of this paper is to use the 3D vector mechanics so that forces can all be represented by global vectors in $X, Y$ \& $Z$ direction and henceforth are easy to study. By doing so the intermittent calculations, as well as the end results, are efficient, coherent and scalable (to any number of a joint like T-joint, or combination of bends and joints). The formulae and procedures are developed with consideration of developing a well-organized CAD-based software for an even better analysis of a variety of irregular shapes in the future with reduced human efforts.
\end{abstract}

\section{Keywords}

Anchor Blocks, Hydropower, Pipelines, Vector Mechanics

\section{Introduction}

All types of above-ground penstock are secured in place at points by anchor blocks. Anchor blocks are the structures which represents the fixed supports of the penstock and are located at vertical or horizontal bends (Mosonyi, 1991). In reality however, there aren't just the cases of individual horizontal and vertical bend but the mixture of both, specially due to the difficult terrains and budget problems in case of Nepal.

The pipelines mainly considered on this paper are the high pressure or low pressure penstocks of hydro-power water conveyance systems, but will also be applicable to analogous systems. The main forces on the anchor blocks are from the dead weights, hydro-static forces, the earth pressures and seismic forces(if considered). There are many other minor forces, which are considered here although a good enough result can be obtained with only the major ones. Although some optimization has been done to get empirical equations(Al-Gahtani, 2009), the 3D approach for the analysis has been rare. Sundberg (2013) has taken 3D approach to calculate the co-ordinates and forces which is based on ASCE (2012). But the vectors are defined only locally and used for determining the important co-ordinates of Anchor Block; so expanding the use of those vectors to calculation of forces, we can avoid the need to resolve the forces in any planes.

\section{Materials and Methods}

The basic definition of the forces which acts on the Anchor Block as per guidelines (ASCE, 2012, Kisan et al., 1984) were used to developed their formula in 3D. Then the design and analysis steps were then modified to accommodate the new formulae.

The formula and derivations in this paper are based on these premises.

1. Forces are transferred from pipe to the anchor blocks and then to the ground.

2. Other supports besides the anchor blocks are the saddle supports for exposed pipelines and the earth itself for the buried ones. 
3. The radius of curvature of the pipe is very small compared to the head of water in the bend, so the head can be assumed constant over the length of a bend.

4. The diameter of pipe is very small compared to the head of water, so the water pressure can be assumed constant over the cross section. Thus, the net resultant of pressure is at the geometric center.

5. The friction force taken for the calculations is the maximum amount which can develop, the friction force on itself can never exceed the total of sliding forces and acts on the opposite direction of the strain produced.

6. The water hammer effect is considered as added dynamic head to the static head, the temporal variation of the force is not considered.

These assumptions will help simplify the calculation of magnitude of the forces associated with the Anchor Blocks, as for the direction of the force vectors we need a way to represent their direction in a more unified way.

\subsection{Co-ordinate Systems}

Since the direction of forces developed in anchor blocks depends upon the directional alignment of the pipes and blocks, two local co-ordinate systems are defined in terms of the global system to make all those forces compatible with one another. The definition of the forces are in local scope, the local system defined here will be used to convert those forces into global ones.

The forces are expressed in terms of the global co-ordinate systems of $\hat{i}, \hat{j}$ and $\hat{k}$ which are the unit vectors along the direction of $\mathrm{X}, \mathrm{Y}$ and $\mathrm{Z}$ axis respectively. Every force consists of three components and can be expressed as $F=F_{X} \hat{i}+F_{Y} \hat{j}+F_{Z} \hat{k}$. The Easting, Northing and Elevation are taken as the $\mathrm{X}, \mathrm{Y}$ and $\mathrm{Z}$ co-ordinates respectively. Using of any projection system will have satisfactory result although mUTM (modified UTM) co-ordinate system with Everest Nagarkot datum is used for the examples.

First local Co-ordinate system written from here onward as Local Co-ordinate system-I has three mutually perpendicular axes one along the flow direction of the water $\left(\hat{r}_{1}\right)$, another perpendicular to the first one and lying completely in the vertical plane passing through the pipe $\left(\hat{r_{2}}\right)$ and the last one perpendicular to the both-that would be left of the pipe horizontally $\left(\hat{r_{3}}\right)$. The two vectors $\hat{r_{1}} \& \hat{r_{2}}$ lie perpendicularly in the vertical plane.

Second local Co-ordinate system written from here onward as Local Co-ordinate system-II consists of one unit vectors each from Global and First Local System which are the unit vector along positive $\mathrm{Z}$ axis $(\hat{k})$ and unit vector perpendicular to the pipe in the horizontal plane $\left(\hat{r_{3}}\right)$, the new unit vector is the unit vector along the direction of the pipe in the horizontal plane $\left(\hat{r_{p}}\right)$. The two vectors $\hat{r_{3}} \& \hat{r_{p}}$ lie perpendicularly in horizontal plane.

The directions of these vectors are illustrated in figures $2 \& 3$.

Representing a force's $\hat{r}_{1}$ component similarly as representing $X$ component of force $F$ as $\vec{F}_{X}$ would cause problems and some unaesthetic symbols, so for simplicity, let's define a vector operation $*$ which gives the vector component of first operand to the second operand. Mathematically it can be expressed as,

$$
\vec{A} * \vec{B}=(\vec{A} \cdot \vec{B}) \vec{B}
$$

As shown in figure 1,when the second operand is a unit vector this operation results in the rectangular component of the first operand to the second $(\vec{F} * \hat{i}$ is same as $\vec{F}_{X}$ ).

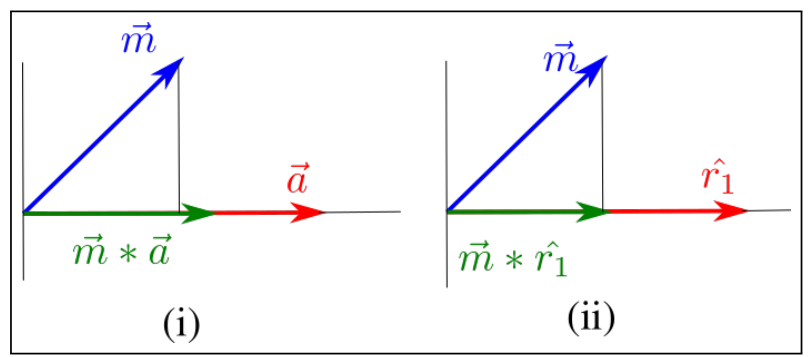

Figure 1: Demonstration of * operator

Hence, the direction of the given expression is always the direction of vector given after $*$ operator.

Conversion of Local Systems in to Global System can be done by considering a pipe with center point spanning between two Point of Intersections(PI) $P I_{1}\left(x_{1}, y_{1}, z_{1}\right)$ and $P I_{2}\left(x_{2}, y_{2}, z_{2}\right)$. The relationships between aforementioned vectors can be calculated using trigonometry and co-ordinate geometry :

The $\hat{r_{1}}$ vector along the flow direction is the displacement vector from start to end of the 
pipe(center point). Hence, it is given as unit direction of displacement vector from $P I_{1}$ to $P I_{2}$.

$$
\hat{r_{1}}=\frac{x_{2}-x_{1}}{l} \hat{i}+\frac{y_{2}-y_{1}}{l} \hat{j}+\frac{z_{2}-z_{1}}{l} \hat{k}
$$

Similarly, $\hat{r_{p}} \& \hat{r_{2}}$ can be calculated as per their definitions.

$$
\begin{aligned}
\hat{r_{p}} & =\frac{x_{2}-x_{1}}{l_{p}} \hat{i}+\frac{y_{2}-y_{1}}{l_{p}} \hat{j}+0 \hat{k} \\
\hat{r_{2}} & =\sin (\alpha) \hat{r_{p}}-\cos (\alpha) \hat{k} \\
& =\left(\hat{k} \times \hat{r_{p}}\right) \times \hat{r_{1}} \\
\hat{r_{3}} & =\hat{r_{1}} \times \hat{r_{2}}=\hat{k} \times \hat{r_{p}}
\end{aligned}
$$

Where $l \& l_{p}$ are the total length and length in plan of the pipe segment. Which can be calculated as;

$$
\begin{aligned}
& l=\sqrt{\left(x_{2}-x_{1}\right)^{2}+\left(y_{2}-y_{1}\right)^{2}+\left(z_{2}-z_{1}\right)^{2}} \\
& l_{p}=\sqrt{\left(x_{2}-x_{1}\right)^{2}+\left(y_{2}-y_{1}\right)^{2}}
\end{aligned}
$$

And $\alpha$ is the angle made by flow direction to the horizontal plane.

$$
\alpha=\arctan \left(\frac{z_{2}-z_{1}}{l_{p}}\right)
$$

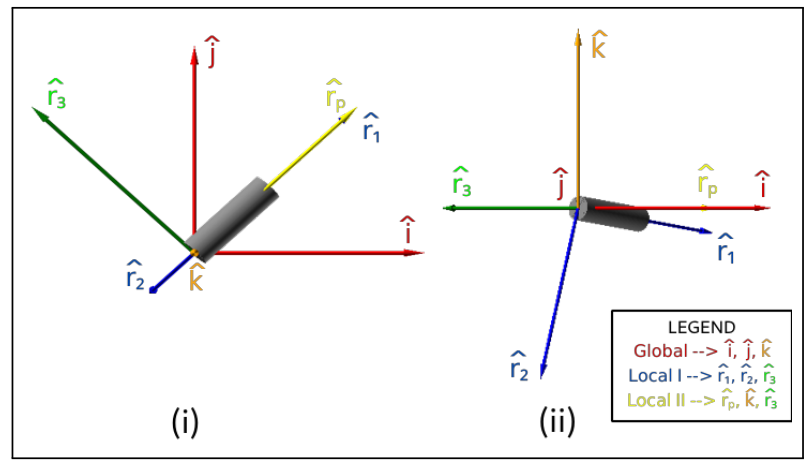

Figure 2: $X Y(i) \& Y Z(i i)$ projections of different axes

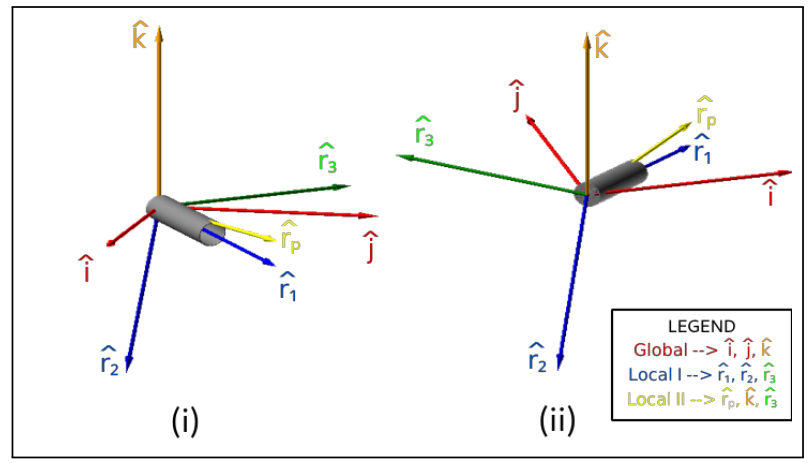

Figure 3: Isometric views of different axes
For the moment calculation, the point of application of forces are also required. The most used Points are:

$$
\begin{aligned}
P_{j}\left(x_{j}, y_{j}, z_{j}\right) & \text { PI of bend } \\
P_{j u}\left(x_{j u}, y_{j u}, z_{j u}\right) & \text { u/s PI of bend } \\
P_{j d}\left(x_{j d}, y_{j d}, z_{j d}\right) & \mathrm{d} / \mathrm{s} \text { PI of bend } \\
P_{i}\left(x_{i}, y_{i}, z_{i}\right) & \text { Center of toe of } i^{t h} \\
& \text { face of Anchor Block } \\
P_{C G}\left(x_{C G}, y_{C G}, z_{C G}\right) & \text { CG of Anchor Block }
\end{aligned}
$$

Other points can be easily determined using geometric rules if need arises.

\subsection{Forces on Anchor Block}

The Anchor blocks' main function is to provide stability from forces in the pipe and environment. It experiences a variety of forces from water inside the pipe, pipe itself, earth surrounding it and the water in the earth. Some of these forces are Major Forces having significant part in resultant, while others can be ignored for approximation. Some forces might be major in some conditions and insignificant in other.

\subsubsection{Self-Weight}

The weight of the components like Pipe, Water and Block are all in $-\hat{k}$ direction, but only their components in the direction of interests are evaluated:

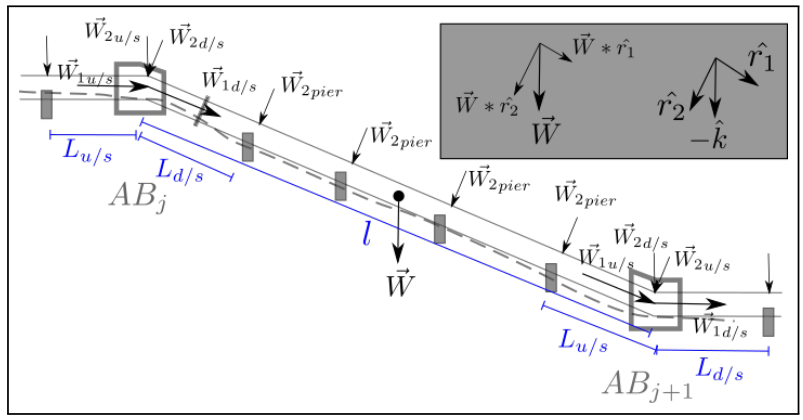

Figure 4: Self-weight of pipe and its components

As shown in figure 4 , the weight of the pipe can be resolved in two direction. Self-weight of pipe material along the pipe axis is calculated by taking the component of weight in $\hat{r}_{1}$ direction i.e. $W_{1}=\vec{W} * \hat{r_{1}}$. This force is equally divided between the two anchor blocks at both end of the pipe.

$$
\vec{W}_{1}=\gamma_{p} \frac{l}{2}(-\hat{k}) * \hat{r_{1}}
$$


Only unit weight of pipe $\left(\gamma_{p}\right)$ is taken as water is free to move in $r_{1}$ direction. $\vec{W}_{1 u / s} \& \vec{W}_{1 d / s}$ are calculated with respective $\hat{r_{1}} \& l$.

Similarly, self-weight of pipe and water perpendicular to the pipe axis is the component along $\hat{r_{2}}$ direction i.e. $W_{1}=\vec{W} * \hat{r_{2}}$. But a major difference except the direction is that the perpendicular component is shared among the saddle supports so only the length between the Block and nearest saddle is used for $F_{\text {weight }}$ unlike in equation 9 where total length between PI is taken. The reason being that this component of weight is distributed between the piers along the pipe length as well asthe Anchor Blocks at the ends.

$$
\vec{W}_{2}=\gamma_{p+w} \frac{L}{2}(-\hat{k}) * \hat{r_{2}}
$$

Again, $\vec{W}_{2 u / s} \& \vec{W}_{2 d / s}$ are calculated with respective $\hat{r_{2}} \& L$.

Self-weight of Anchor Block is used for the stability analysis, it provides most amount of stabilizing force and moment. It can be calculated as unit weight multiplied by volume of block. Summation of different parts can be done if it consists of more than one material.

$$
\vec{W}_{B}=-\gamma_{\text {conc }} V_{B} \hat{k}
$$

\subsubsection{Hydro-static Forces}

Hydro-static forces in penstock that we need to consider are due to bend and reducer. Same principle can be applied in case of other type of joints. In a straight part of pipe, the hydro-static forces of water will be cancelled out in the direction of flow. The remaining hydro-static force is radially outward. That force will be balanced by hook's stress of pipe and has zero resultant.

But in case of bend, the force on flow direction doesn't get canceled out due to change in direction, it can be determined by taking the integration of all the hydro-static forces in the length of bend. Which yields,

$$
\vec{H}_{b}=\rho_{w} g Z A\left(\hat{r}_{1 u / s}-\hat{r}_{1 / s}\right)
$$

The sign convention is $+v e$ for incoming and $-v e$ for outgoing pipes, since a bend has two pipes a simple vector difference will suffice.

Similarly in case of reducer, the change in area in entry and exit yields some residual hydro-static force given by,

$$
\vec{H}_{r}=\rho_{w} g Z\left(A_{u / s}-A_{d / s}\right)\left(\hat{r}_{1 u / s} O R \hat{r}_{1 / / s}\right)
$$

The variation in pressure throughout the pipe section and length (Munson et al., 2009) are considered to be insignificant in case of huge head $(Z)$.

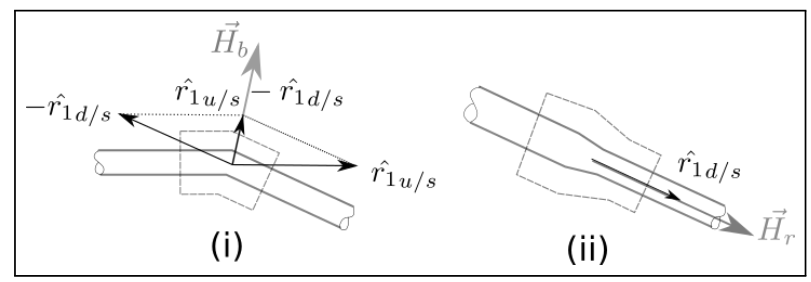

Figure 5: Directions of Hydrostatic Forces on horizontal bend (i) and reducer (ii)

\subsubsection{Seismic Forces}

Seismic forces are the forces developed due to relative movements between the block and the earth. As Nepal has been vulnerable to Earth quakes due to its geological structures(Pandey et al., 1995), and has had recent event of major earthquake in 2015 (Bhattarai et al., 2015), the seismic forces are major forces in design/analysis of any structure.

The horizontal seismic force is calculated by taking the multiplication of weight by horizontal seismic coefficient.

$$
S_{H}=K_{H} W_{B}
$$

The direction of horizontal is unknown till the point of impact, so for critical condition the direction can be taken as the direction of resultant force to produce the maximum sliding force.

Similarly, vertical seismic force is the multiplication with vertical seismic coefficient. The direction of force can be taken upward for critical condition as downward force would increase stability.

$$
\overrightarrow{S_{V}}=K_{V} W_{B}( \pm \hat{k})
$$

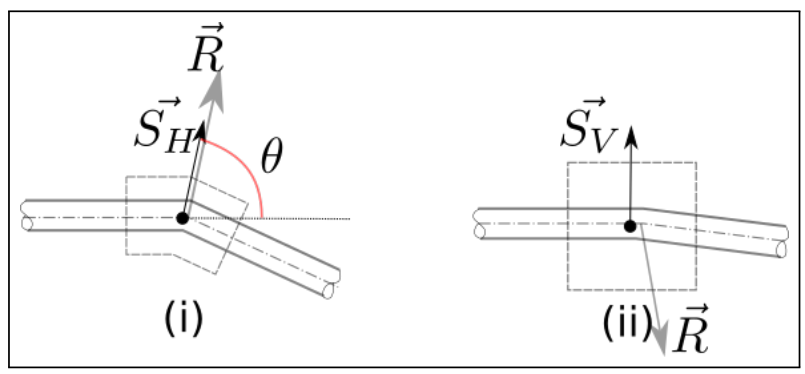

Figure 6: Seismic forces in plan (i) and section along centerline (ii) 
In figure 6 the $\theta$ angle is arbitrary as the seismic force can act on any direction at the time of earthquake and hence for analysis it should be taken in a direction that is most critical to the structure, for now that is the same direction of resultant as it'd maximize the sliding force. Also, the point of application of seismic force is the Center of Gravity of the Anchor Block, the point of application of resultant may be different.

\subsubsection{Force from Earth Pressures}

Earth pressure is unavoidable since the blocks rest on the earth itself. In certain conditions the pressure from the earth can help establish the block but sometimes it can be major force in destabilizing. Since forces due to earth pressures are more in opposite direction of displacement, most of the time it is resisting in nature. The earth pressure is directly dependent on the depth of the soil, hence its distribution is triangular (Arora, 1987). The total force due to soil of depth $h$ can be calculated by equation 16 . This force acts at a point $1 / 3^{\text {rd }}$ from the bottom vertically and at the center of face horizontally. This force acts on each face of the block, the force on the $i^{\text {th }}$ face due to the soil touching it can be calculated as:

$$
\vec{E}_{i}=\frac{1}{2} k \gamma_{s} l_{i} h^{2}\left(-\hat{n}_{i}\right)
$$

Here, the unit vector $\hat{n}_{i}$ is perpendicular to the face of the block on which the pressure is acting. The direction of unit vector is outward from the surface of the block as shown in figure 7(ii).

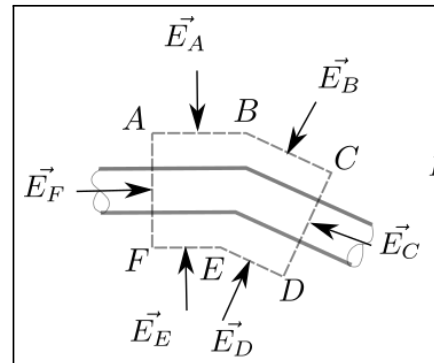

(i)

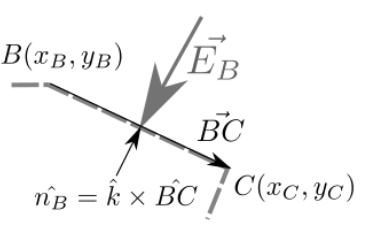

(ii)
Figure 7: Direction of forces due to earth pressure

In the case that the soil is saturated, submerged unit weight of soil $\left(\gamma_{s}^{\prime}\right)$ is used instead of $\left(\gamma_{s}\right)$.

Here $k$ is the coefficient of earth pressure which depends on the direction of movement of block. If $R$ is the resultant force causing the displacement,

$$
k= \begin{cases}k_{a} & \text { for }\left(\vec{R} \cdot \hat{n}_{i}\right)<0 \\ k_{p} & \text { for }\left(\vec{R} \cdot \hat{n}_{i}\right)>0 \\ k_{0} & \text { for }\left(\vec{R} \cdot \hat{n}_{i}\right)=0\end{cases}
$$

Since we don't want the block to actually start sliding (which means stability-failure) at-rest coefficient of earth pressure $\left(k_{0}\right)$ instead of passive earth pressure $\left(k_{p}\right)$ is used in design for $\left(\vec{R} \cdot \hat{n}_{i}\right) \geq 0$.

\subsubsection{Ground Water Up-thrust}

When the ground is saturated, it not only reduces the Coefficient of friction, it can also give the up-thrust which helps reduce the vertical component of resultant. This force doesn't need to be considered if the site has good drainage.

And the Upthrust force on the base in that condition can be calculated as the weight of the water displaced, i.e. if the volume of block under the ground water level (shaded part in figure 8) is $V$ then the upthrust $U$ is equal to $\gamma_{w} V$ (Munson et al., 2009) acting on the $\mathrm{CG}$ of the displaced water in $\hat{k}$ direction.

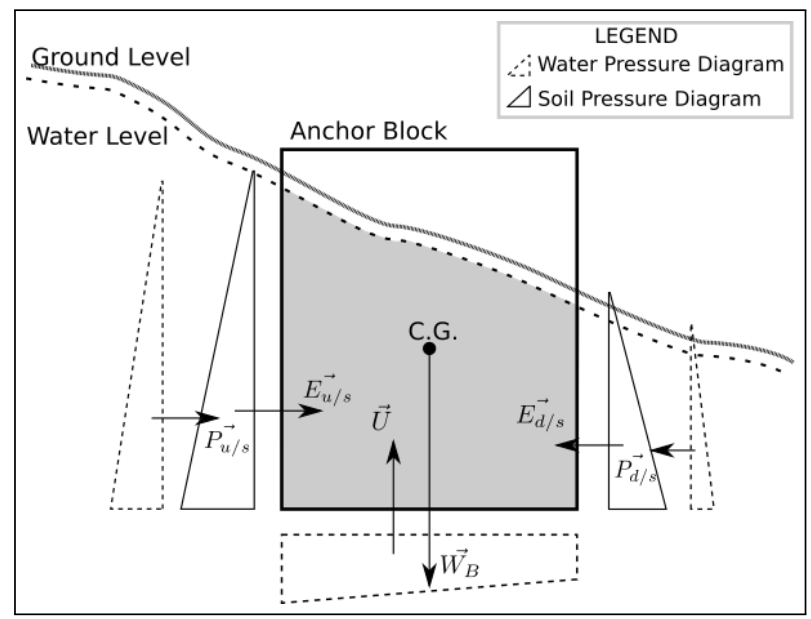

Figure 8: Ground Water Forces acting on a Block (forces on only $\mathrm{u} / \mathrm{s}$ and $\mathrm{d} / \mathrm{s}$ faces)

Assuming horizontal level of water at height of $h$ from anchor block base, the upthrust force for a block with projected base area of $A$ can be calculated as,

$$
\vec{U}=c \gamma_{w} h A_{\text {base }}(\hat{k})
$$

While, the horizontal water force on $i^{\text {th }}$ face can be calculated with formula for water force (Munson et al., 2009),

$$
\vec{P}_{i}=\frac{1}{2} \gamma_{w} l_{i} * h_{i}^{2}\left(-\hat{n}_{i}\right)
$$

When the water level doesn't vary between the faces of the block the resultant horizontal resultant (vector sum of all forces given by equation 18) is zero. 


\subsubsection{Hydrodynamic Forces}

The forces due to change in the direction of movement of the water is given by the rate of change of the momentum of the water Munson et al. (2009). Same as hydro-static forces hydrodynamic forces are also calculated for two cases.

Hydrodynamic forces in a bend is again the integration of moment change over the span of bend. Which yields,

$$
\vec{H}_{b-d y n}=\rho_{w} Q v\left(\hat{r}_{1 u / s}-\hat{r}_{1 d / s}\right)
$$

Similarly hydrodynamic forces in a reducer is the change in momentum due to velocity change,

$$
\vec{H}_{r-d y n}=\rho_{w} Q\left(v_{u / s} \hat{r}_{1 u / s}-v_{d / s} \hat{r}_{1 / / s}\right)
$$

The direction of these forces are same as the hydrostatic forces, which are illustrated in figure 5.

\subsubsection{Thermal Forces}

Thermal fores are developed due to change in the length of the pipe which in turn is caused by the change in the temperature of the environment. In case of the buried pipes the temperature variation isn't significant, and in case of expansion joints, this force is used in conjunction with the frictional force.

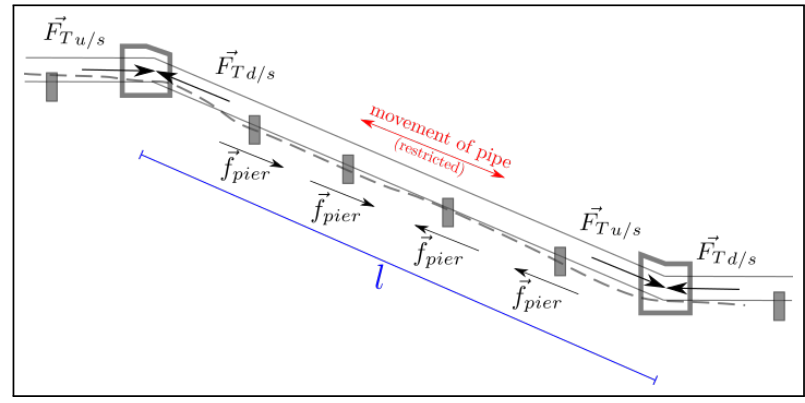

Figure 9: Thermal forces during expansion

$$
\vec{F}_{T}=A_{p} E \Delta T \alpha_{L}\left(\hat{r}_{1 u / s}-\hat{r}_{1 d / s}\right)
$$

Here, $A_{p}=\pi D t$ is the cross-sectional area of pipe material.

Although thermal forces will be nullified at expansion joints, the friction resisting the transfer of the strain to the expansion joint will make the direction of friction force opposite to the strain from temperature change. If no expansion joints are provided thermal forces act directly to the blocks at the two ends (friction force saddles help to distribute the force as shown in figure 9). But the friction force can be neglected as it is only resistive in nature and the thermal force is considered to act directly on the Blocks. But in the case that Expansion joint is provided the Thermal force is neutralized at the joint, but the frictional force resists the neutralization and becomes important.

\subsubsection{Frictional Forces}

Since frictional force is resistive in nature it mostly acts to help the stability by decreasing other forces. For example if only self weight of pipe was to act on it, the friction along the piers will act upwards to resist the pipe sliding downwards from self weight. So, in such cases this force can be neglected.

It becomes a major destablizing force when expansion joints are provided because it resists the release of thermal force on expansion joints.

In case of pipes with expansion joints, the stress due to expansion/contraction is canceled out only if it travels to the expansion joint, so the friction with the saddle can prevent the force transfer and add that force to Anchor Block.

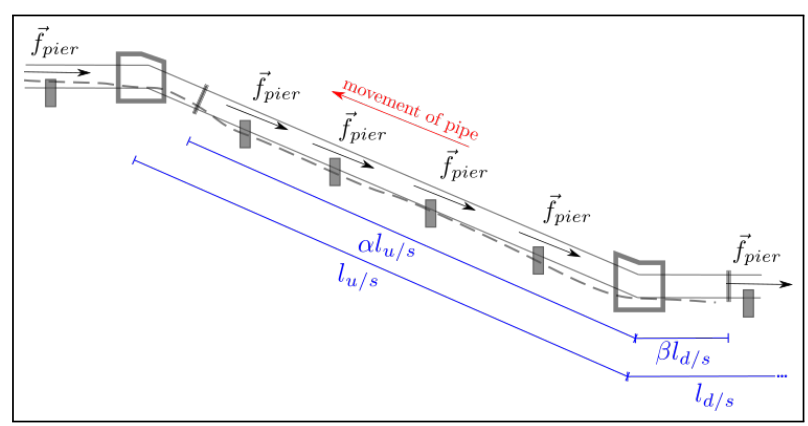

Figure 10: Frictional forces

In such case the frictional force is the minimum of thermal forces developed and maximum frictional resistance. While in case without expansion joint this force can help reduce the transfer of thermal force into the Anchor Blocks, but that condition is not considered as it favors the stability.

The maximum amount of resisting force a single pier can provide in this condition can be calculated as,

$$
\overrightarrow{f_{\text {max }}}=\mu_{s} \gamma_{p+w}\left(L_{\text {pier }} \hat{r_{2}} \cdot \hat{k}\right) \hat{r_{1}}
$$

Here, $L_{\text {pier }}$ is the distance between the piers, and the total frictional force can be found out by multiplying this with number of piers.

For friction with soil where piers are not present, the 
frictional force can be calculated by,

$$
\begin{aligned}
& \overrightarrow{f_{\max }} \\
& \quad=\gamma_{p+w} \mu\left(\alpha L_{u / s}\left(\hat{r}_{2 u / s} \cdot \hat{k}\right) \hat{r}_{1 u / s}+\beta L_{d / s}\left(\hat{r}_{2 / s} \cdot \hat{k}\right) \hat{r}_{d / s}\right)
\end{aligned}
$$

Where, $\alpha$ and $\beta$ are coefficients ranging from -1 to +1 ; their sign are $+v e$ and -ve according to the direction of movement of pipe respective to saddle. And their magnitude depends on whether the expansion joints are present or not. If expansion joints are not provided take $\alpha=\beta=0.5$; otherwise, take the coefficients as the ratio of distance to the expansion joint to the total distance to the next anchor block in respective sides (shown in figure 10).

It is important to note that, these values are maximum amount of friction force and actual friction force can never surpass the force it is resisting. So, the actual friction force can not exceed the value of Thermal Force if expansion joint was absent (as given from equation (21)).

\subsubsection{Provision for Other Forces}

Extra forces (like force on rock anchorages) for special conditions can be added as a vector as specified in the procedure below, the point of action and the vector form of the force has to be developed before proceeding. Because of that, the characteristics of the force has to be known before adding it to this model.

\subsubsection{Calculation of Resultant}

The resultant of the forces is simply the vector sum of the forces acting on the anchor block. The line of action of the resultant can be found by adding the moments of all the forces about any axis of rotation, then use equation 28. The forces are classified as Block Independent, Block Dependent and Displacement Dependent (labeled respectively as BI, BD \& DD in Table 1) for the resultant calculation procedure.

For precision analysis all forces are to be considered while for rough estimation only considering major forces will result in practical enough result in most cases.

1. Add the Block Independent forces to get the first resultant

$$
\vec{R}_{1}=\vec{W}_{1}+\vec{W}_{2}+\vec{H}_{b}+\vec{H}_{r}+\overrightarrow{H_{d y n}}
$$

Table 1: Classification of Forces

\begin{tabular}{|l|c|l|l|}
\hline Forces & BI & BD & DD \\
\hline \multicolumn{4}{|c|}{ Major Forces } \\
\hline Self-Weight & $\checkmark$ & $\checkmark$ & \\
\hline Hydro-static Forces & $\checkmark$ & & \\
\hline Seismic Forces & & & $\checkmark$ \\
\hline Earth Forces & & & $\checkmark$ \\
\hline Ground Water Upthrust & & $\checkmark$ & \\
\hline \multicolumn{4}{|c|}{ Minor Forces } \\
\hline Frictional Force & & & $\checkmark$ \\
\hline Hydrodynamic Forces & $\checkmark$ & & \\
\hline Thermal forces & & & $\checkmark$ \\
\hline Other Forces & \multicolumn{3}{|c|}{ Undefined } \\
\hline
\end{tabular}

2. Assume a Block Shape \& Size

3. Calculate Block Dependent forces

4. Calculate the value of $\alpha$ and $\beta$ then the value of frictional force.

5. Calculate second resultant as:

$$
\vec{R}_{2}=\vec{R}_{1}+\vec{W}_{B} \pm \vec{f}
$$

add $\vec{F}_{T}$ to equation 25 if no expansion joints are present.

Note: Use + for expansion case and - for contraction case.

6. Use $\vec{R}_{2}$ as resultant and calculate the forces due to earth pressure.

7. Add the earth forces to the resultant, and if there are any special forces add them too.

$$
\vec{R}=\vec{R}_{2}+\sum \vec{E}_{i}+f_{\text {special }}
$$

8. Calculate the Seismic forces and upthrust if required, but don't add it to the resultant.

\subsubsection{Output of the Calculation}

The force calculation steps will yield the resultant force in vector form $\left(\vec{R}=R_{x} \hat{i}+R_{y} \hat{j}+R_{z} \hat{k}\right)$ as well as its line of action. The Seismic forces and Ground water up thrust will also be available to combine with the resultant in their respective cases of analysis.

The resultant force's line of action can be determined by Varignon's theorem given in Varignon (n.d.). Considering any point $O\left(x_{0}, y_{0}, z_{0}\right)$ in a rotation plane; 
we can calculate the moment of all the forces around the rotation axis as:

$$
\vec{M}=\sum \vec{r}_{i} \times \vec{F}_{i}=\vec{r} \times \vec{R}
$$

Here, $\vec{r}_{i}=P_{i}-O$ where $r_{i}, P_{i} \& F_{i}$ are the moment arm, point of action of $i^{\text {th }}$ force and that force respectively, while $\vec{r}$ and $\vec{R}$ are lever arm of resultant and the resultant. If $\mathrm{r}$ is the perpendicular distance from $\mathrm{O}$ to the line of action of the force,

$$
r=\frac{|\vec{M}|}{|\vec{R}|}
$$

\subsection{Stability Analysis}

After the calculation of all the forces, the stability of the block can be calculated in different cases using many parameters. The cases considered for the stability analysis are Expansion case (E), Contraction case (C), Seismic case (S) and Ground water saturated case $(\mathrm{G})$. Other cases can also be used for analysis if conditions and combination of forces are known for that case, usually it's superfluous to analysis for non-critical cases.

The numerical parameters calculated for determining the safety are, Factor of Safety against Sliding, Overturning, eccentricity and the pressure at the base of the block. The factor of safety against sliding in a sliding direction $\hat{s}$ is calculated as,

$$
F o S=\mu \frac{\vec{R} \cdot \hat{p}}{\vec{R} \cdot \hat{s}}
$$

Here, $R$ is the resultant force of all the forces on the block, and $\hat{p}$ is the unit vector perpendicular to the sliding plane(towards the plane).

For horizontal sliding plane, the minimum factor of safety is when the direction of slide is same as the horizontal direction of resultant. So equation 29 becomes,

$$
F o S_{\text {min }}=\mu \frac{-R_{z}}{\sqrt{\left(R_{x}\right)^{2}+\left(R_{y}\right)^{2}}}
$$

Here in equation $29 \& 30, \vec{R}=R_{x} \hat{i}+R_{y} \hat{j}+R_{z} \hat{k}$ is the total resultant force.

When seismic conditions are considered, $S_{H}$ is added to the denominator and $S_{V}$ is subtracted from numerator (in a way that results in least $F o S$ ). And when Ground water is considered, $U$ is subtracted from the numerator (value of $\mu$ is also likely to change)
Now, to analyze the safety against overturning, the moment of all the forces are calculated with the formula,

$$
\vec{M}=\vec{r} \times \vec{F}
$$

Where, $r$ is the vector joining the point of rotation and the force $(F)$ in the rotating plane. Taking a unit vector pointing out from the rotating plane $\hat{m}$ in the same direction as overturning rotation axis, Factor of safety which is defined as the ratio of resisting moment to overturning moment (Arora, 1987):

$$
F o S=-\frac{\sum_{-v e} \vec{M} \cdot \hat{m}}{\sum_{+v e} \vec{M} \cdot \hat{m}}
$$

Where the sum of positive represents overturning moment, while negative is stabilizing moment.

When seismic conditions are considered, effects of $S_{H} \& S_{V}$ are taken as they act at CG of block. Their direction is again taken in such a way that they produce maximum overturning moment.

When ground water is considered, effect of $U$ is added considering it acts on CG of base.

The check for eccentricity is that the line of action should pass through the middle third of the base of the block.

The intersection of line of action of the resultant with the base of the Block is the point of action of the force in the base. Now eccentricity can be found out by determining the horizontal distance between the point of action and the center of the base. This eccentricity is then used to check the bearing pressures at each vertex of base. The bearing pressures should lie between zero and Bearing capacity of the soil.

\section{Results and Discussions}

The formulae and procedures for the Design and Analysis of the Anchor Blocks are derived using the vector approach. Those formulae were verified by converting them to the trigonometric formulae in a $2 \mathrm{~d}$ setting. The trigonometric formulae for the analysis can be found in many guidelines and are easier to be verified.

\subsection{Verification Using Trigonometric Method}

As trigonometric method is little complicated for 3D, we can assume $\mathrm{Y}$ co-ordinates of the pipe to be same for comparison, so there is only vertical deflection. 
The formulae for the unit vectors in 2.1 will have $\left(y_{2}-\right.$ $\left.y_{1}\right)=0$. The equation 2 becomes,

$$
\hat{r} 1=\frac{x_{2}-x_{1}}{l} \hat{i}+\frac{z_{2}-z_{1}}{l} \hat{k}
$$

Similarly, $\hat{r_{p}}$ is just $\hat{i}$ and $\hat{r_{3}}$ is $\hat{j}$. While, $\hat{r_{2}}$ is still $\hat{j} \times \hat{r_{1}}$.

Now, if we use vertical deflection angle $\alpha$ to convert co-ordinate differences to trigonometric functions. We'll have,

$$
\begin{aligned}
\tan (\alpha) & =\frac{z_{2}-z_{1}}{x_{2}-x_{1}} \\
\sin (\alpha) & =\frac{z_{2}-z_{1}}{l} \\
\cos (\alpha) & =\frac{x_{2}-x_{1}}{l}
\end{aligned}
$$

Where, $l=\sqrt{\left(x_{2}-x_{1}\right)^{2}+\left(z_{2}-z_{1}\right)^{2}}$ is the length between the PI. Now, redefining the $\hat{r_{1}}$ vector:

$$
\hat{r_{1}}=\cos \alpha \hat{i}+\sin \alpha \hat{k}
$$

And, $\hat{r_{2}}$ vector:

$$
\hat{r_{2}}=\sin \alpha \hat{i}-\cos \alpha \hat{k}
$$

Let's assume $\left(\alpha_{u / s}, \alpha_{d / s}\right)$ as $(\alpha, \beta)$ in this section for simplicity.

Now replacing the values of the unit vectors in 2 dimension in the formulae of forces from 2.2Forces on Anchor Block.

Replacing the values of unit vectors in Self-Weight forces from section-2.2.1 we can get the trigonometric formulae as given in Kisan et al. (1984) and DOED Design Guidelines: Water Conveyance System of Hydropower Projects (2007).

Equation 9 after substitution:

$$
\begin{aligned}
\vec{W}_{1} & =\gamma_{p} \frac{l}{2}(-\hat{k}) \cdot(\cos \alpha \hat{i}+\sin \alpha \hat{k}) \hat{r}_{1} \\
& =-\gamma_{p} \frac{l}{2} \sin \alpha \hat{r_{1}}
\end{aligned}
$$

So, the self weights along the flow direction due to upstream and downstream pipe segment can be expressed as:

$$
\begin{aligned}
W_{1 u / s} & =\gamma_{p} \frac{l_{u / s}}{2} \sin \alpha \\
W_{1 d / s} & =\gamma_{p} \frac{l_{d / s}}{2} \sin \beta
\end{aligned}
$$

Similarly, equation 10 becomes:

$$
\begin{aligned}
\vec{W}_{2} & =\gamma_{p+w} \frac{L}{2}(-\hat{k}) \cdot(\sin \alpha \hat{i}-\cos \alpha \hat{k}) \hat{r}_{2} \\
& =-\gamma_{p+w} \frac{L}{2} \cos \alpha * \hat{r_{2}}
\end{aligned}
$$

$W_{2}$ due to upstream and downstream pipe segments are now:

$$
\begin{aligned}
& W_{2 u / s}=\gamma_{p+w} \frac{L_{u / s}}{2} \cos \alpha \\
& W_{2 d / s}=\gamma_{p+w} \frac{L_{d / s}}{2} \cos \beta
\end{aligned}
$$

The self weight of Anchor Block $W_{B}$ is simply $\gamma_{c o n c} V_{B}$, which doesn't need conversion.

Hydrostatic Forces The forces from section 2.2.2 can also be converted in similar manner. The hydrostatic force on bend after replacing the values of unit vectors is:

$$
\vec{H}_{b}=\gamma_{w} Z A(\cos \alpha \hat{i}+\sin \alpha \hat{k}-\cos \beta \hat{i}-\sin \beta \hat{k})
$$

Now, magnitude of the force given by square root of the sum of squares of the components is,

$$
H_{b}=\gamma_{w} Z A \sqrt{(\cos \alpha-\cos \beta)^{2}+(\sin \alpha-\sin \beta)^{2}}
$$

Now, expanding and replacing $\cos ^{2} \theta+\sin ^{2} \theta$ by 1 , we get,

$$
=\gamma_{w} Z A \sqrt{1-2 \cos \alpha \cos \beta+1-2 \sin \alpha \sin \beta}
$$

Now, we know that $\cos (\alpha-\beta)$ $=\cos \alpha \cos \beta+\sin \alpha \sin \beta \& \cos 2 \theta=1-2 \sin ^{2} \theta$,

$$
\begin{aligned}
& =\gamma_{w} Z A \sqrt{2-2 \cos (\alpha-\beta)} \\
& =\gamma_{w} Z A \sqrt{2-2\left(1-2 \sin ^{2}\left(\frac{\alpha-\beta}{2}\right)\right)}
\end{aligned}
$$

Finally, we get the hydro-static force in a bend as:

$$
H_{b}=2 \gamma_{w} Z A \sin \left(\frac{|\alpha-\beta|}{2}\right)
$$

For Hydrodynamic Forces, using the evaluations from equation 43 to 44 we can directly write,

$$
\left|\hat{r}_{1 u / s}-\hat{r}_{1 / s}\right|=2 \sin \left(\frac{|\alpha-\beta|}{2}\right)
$$


Thus, the hydrodynamic force from 2.2.6 can now be redefined as,

$$
H_{d y n}=2 \rho_{w} Q v \sin \left(\frac{|\alpha-\beta|}{2}\right)
$$

replacing the value of $\operatorname{velocity}(v)$ in terms of discharge $(Q)$ \& diameter $(d)$, we get,

$$
H_{d y n}=\rho_{w} \frac{8 Q^{2}}{\pi d^{2}} \sin \left(\frac{|\alpha-\beta|}{2}\right)
$$

As for the frictional forces from section 2.2.8, replacing the unit vectors yields,

$$
\overrightarrow{f_{\text {max }}}=\mu_{s} \gamma_{p+w} L_{\text {pier }}((\sin \alpha \hat{i}-\cos \alpha \hat{k}) \cdot \hat{k}) \hat{r_{1}}
$$

Which evaluates to $\mu_{s} \gamma_{p+w} L_{\text {pier }}(\cos \alpha) * \hat{r_{1}}$, therefore the magnitude of the friction force is,

$$
f_{\text {max }}=\mu_{s} \gamma_{p+w} L_{p i e r}(\cos \alpha)
$$

\subsection{Sample Calculation}

Now, Using the methods described before, a sample calculation was done for a T-joint with a Bend immediately following. Calculation of which would have been very tedious with trigonometric methods. The units of Forces, Moments and Lengths are in $k N$, $k N-m \& m$ respectively.

The main inputs are the hydraulic parameters and the alignment geometry. For a calculation/analysis of a long alignment, only varying the contents of table 3 will give all the block independent forces. The design parameters are shown in table 2, for a project this table mostly remains same, the pipe diameter and the total head may defer for headrace pipe and penstock pipe.

Using the co-ordinates given in table 3 we can calculate the unit vectors in local co-ordinate system for all the pipes, the important ones are: Headrace to Tee(P1), Bend to Penstock(P2), Tee to SurgeTank(P3), their unit vectors are given in table 4 . After the unit vectors are calculated, the hydro-static forces of those pipes can be calculated using the formula given in equation 12. The point of actions for these forces shown in table 5 are respectively Tee, Bend and Tee point. Similarly, all the other forces can be calculated as per section 2.2, and we'll get their vector form as well as point of application.

Now, a shape of Anchor Block is assumed (and iteratively modified till all safety parameters are within satisfactory limit).
Table 2: Design Parameters

\begin{tabular}{|l|c|rl|}
\hline Description & Symbol & Value & \\
\hline Internal Diameter & $D$ & 1200 & $\mathrm{~mm}$ \\
\hline Thickness & $t$ & 8 & $\mathrm{~mm}$ \\
\hline Outer Diameter & $D_{o}$ & 1216 & $\mathrm{~mm}$ \\
\hline Internal Area & $A$ & 1130973 & $\mathrm{~mm}^{2}$ \\
\hline Steel Area & $A_{s}$ & 30360 & $\mathrm{~mm}^{2}$ \\
\hline Steel Density & $\rho_{s}$ & 7.85 & $\mathrm{~kg} / \mathrm{m}^{3}$ \\
\hline Water Density & $\rho_{w}$ & 1 & $\mathrm{~kg} / \mathrm{m}^{3}$ \\
\hline Crest Level & $C L$ & 1409.5 & $\mathrm{amsl}$ \\
\hline Static Head & $Z_{0}$ & 15.85 & $\mathrm{~m}$ \\
\hline Dynamic Head & $Z_{d y n}$ & 20 & $\mathrm{~m}$ \\
\hline Total Head & $Z$ & 35.85 & $\mathrm{~m}$ \\
\hline Weight of Water & $\gamma_{w}$ & 11.09 & $\mathrm{kN} / \mathrm{m}$ \\
\hline Weight of Pipe & $\gamma_{p}$ & 2.34 & $\mathrm{kN} / \mathrm{m}$ \\
\hline Total Weight & $\gamma_{w+p}$ & 13.43 & $\mathrm{kN} / \mathrm{m}$ \\
\hline Soil Density & $\gamma_{s o i l}$ & 18 & $\mathrm{kN} / \mathrm{m}^{3}$ \\
\hline Angle of Friction & $\phi$ & 22.5 & $\circ$ \\
\hline Active Coeff. & $K_{a}$ & 0.4465 & \\
\hline At-rest Coeff. & $K_{0}$ & 0.6173 & \\
\hline Passive Coeff. & $K_{p}$ & 2.2398 & \\
\hline
\end{tabular}

Table 3: Co-ordinates of PI

\begin{tabular}{|l|c|c|c|}
\hline Locations & $\mathrm{X}$ & $\mathrm{Y}$ & $\mathrm{Z}$ \\
\hline headrace & 425818.13 & 3069492.75 & 1394.31 \\
\hline tee $(\mathrm{T})$ & 425792.82 & 3069487.00 & 1393.65 \\
\hline surgetank & 425804.56 & 3069477.34 & 1400.81 \\
\hline bend $(\mathrm{B})$ & 425791.66 & 3069487.95 & 1393.50 \\
\hline penstock & 425779.12 & 3069513.90 & 1390.63 \\
\hline
\end{tabular}

Table 4: Unit Vectors for Pipes

\begin{tabular}{|l|r|r|}
\hline Pipe & $\hat{r_{1}}$ & \multicolumn{1}{|c|}{$\hat{r_{p}}$} \\
\hline P1 & $-0.97 \hat{i}-0.22 \hat{j}-0.03 \hat{k}$ & $-0.98 \hat{i}-0.22 \hat{j}$ \\
\hline P2 & $-0.43 \hat{i}+0.90 \hat{j}-0.10 \hat{k}$ & $-0.44 \hat{i}+0.90 \hat{j}$ \\
\hline P3 & $0.70 \hat{i}-0.57 \hat{j}+0.43 \hat{k}$ & $0.77 \hat{i}-0.64 \hat{j}$ \\
\hline \hline Pipe & $\hat{r_{2}}$ & \multicolumn{1}{r}{$\hat{3}$} \\
\hline P1 & $0.02 \hat{i}+0.01 \hat{j}-1.00 \hat{k}$ & $0.22 \hat{i}-0.97 \hat{j}$ \\
\hline P2 & $0.04 \hat{i}-0.09 \hat{j}-0.99 \hat{k}$ & $-0.90 \hat{i}-0.43 \hat{j}$ \\
\hline P3 & $0.30 \hat{i}-0.24 \hat{j}-0.82 \hat{k}$ & $0.57 \hat{i}+0.70 \hat{j}$ \\
\hline
\end{tabular}


Table 5: Hydro-static Forces

\begin{tabular}{|l|r|r|}
\hline & Value & Vector Form \\
\hline P1 & 175.85 & $-171.43 \hat{i}-38.95 \hat{j}-4.47 \hat{k}$ \\
\hline P2 & 397.75 & $172.21 \hat{i}-356.36 \hat{j}+39.41 \hat{k}$ \\
\hline P3 & 397.75 & $-277.87 \hat{i}+228.64 \hat{j}-169.47 \hat{k}$ \\
\hline Total & 350.22 & $-277.09 \hat{i}-166.67 \hat{j}-134.52 \hat{k}$ \\
\hline
\end{tabular}

Table 6: Anchor Block Geometry

\begin{tabular}{|c|r|r|r|}
\hline Start Pt. & $\mathrm{X}$ & $\mathrm{Y}$ & Length \\
\hline face A & 425794.78 & 3069488.60 & 2.26 \\
\hline face B & 425795.28 & 3069486.40 & 2.20 \\
\hline face C & 425793.88 & 3069484.69 & 3.08 \\
\hline face D & 425791.50 & 3069486.65 & 1.33 \\
\hline face E & 425790.61 & 3069487.65 & 1.32 \\
\hline face F & 425790.01 & 3069488.82 & 2.22 \\
\hline face G & 425792.00 & 3069489.79 & 1.90 \\
\hline face H & 425793.08 & 3069488.22 & 1.74 \\
\hline
\end{tabular}

This co-ordinates shown in table 6 describe the shape as seen in figure 12. Here the height of the block also varies between the edges (not shown in figure). The volume of Block was $49.38 \mathrm{~m}^{3}$, CG at $(425792.942,3069487.2734,1394.0166)$ and total weight of Block including water and pipe directly inside it comes out to be $-1116.6 \vec{k}$. All of these measurements are taken from a CAD software.

With these co-ordinates of the points, we can calculate the directions of all the faces, which will give the rotational axis and direction of earth pressures. For example, the vectors along the face- $\mathrm{A}$ and perpendicular(outward) to the face-A are .

The earth pressure can be calculated after we have the block geometry and the direction of the resultant of block independent forces. The calculated earth pressures are shown in table 7 , the method to determine whether to use active or at rest coefficient was explained while defining the equation (16).

All the forces as shown in table 5 and 7 are plotted in the figure 11 and 12 for ease of visualization.

As we can see, the Sum of all 3 hydrostatic forces is used to determine the coefficient for earth pressure. If the final resultant's direction deviates from this significantly next iteration can be done with new coefficients of earth pressure.
Table 7: Forces due to Earth Pressures

\begin{tabular}{|c|l|r|c|}
\hline Face & $K$ & Force & Vector Form \\
\hline $\mathrm{A}$ & $K_{a}$ & 145.31 & $-141.70 \hat{i}-32.20 \hat{j}$ \\
\hline $\mathrm{B}$ & $K_{a}$ & 141.45 & $-109.45 \hat{i}+89.61 \hat{j}$ \\
\hline $\mathrm{C}$ & $K_{0}$ & 273.78 & $174.05 \hat{i}+211.34 \hat{j}$ \\
\hline $\mathrm{D}$ & $K_{0}$ & 118.23 & $88.31 \hat{i}+78.60 \hat{j}$ \\
\hline $\mathrm{E}$ & $K_{0}$ & 117.34 & $104.41 \hat{i}+53.54 \hat{j}$ \\
\hline $\mathrm{F}$ & $K_{a}$ & 142.74 & $62.54 \hat{i}-128.31 \hat{j}$ \\
\hline $\mathrm{G}$ & $K_{a}$ & 122.16 & $-100.65 \hat{i}-69.24 \hat{j}$ \\
\hline $\mathrm{H}$ & $K_{a}$ & 111.88 & $24.41 \hat{i}-109.18 \hat{j}$ \\
\hline Total & & 138.76 & $101.92 \hat{i}+94.17 \hat{j}$ \\
\hline
\end{tabular}

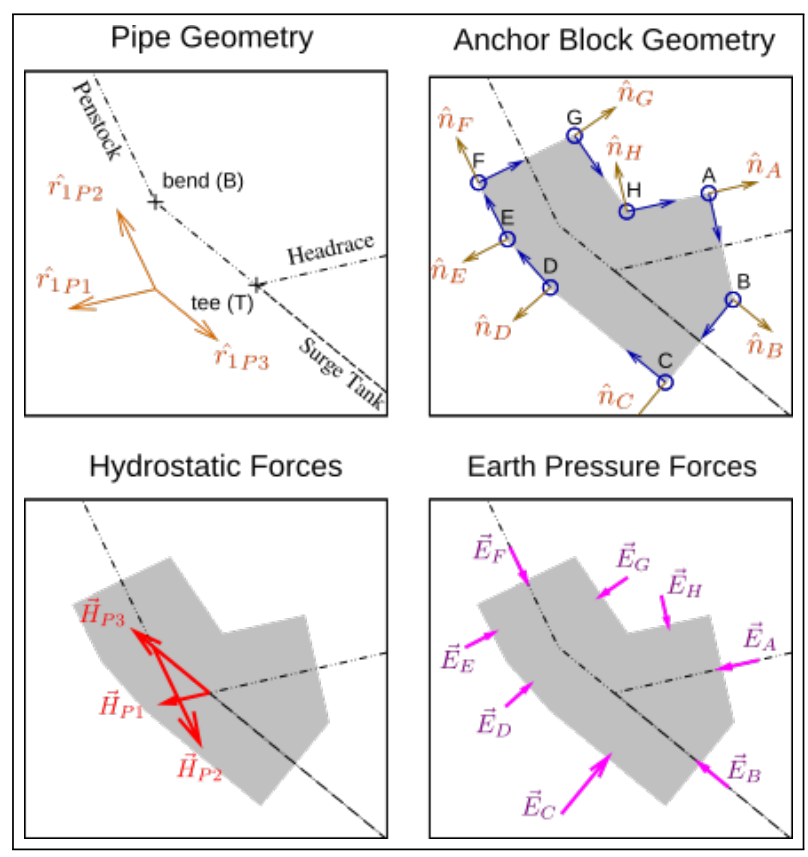

Figure 11: Various Forces and Unit Vectors

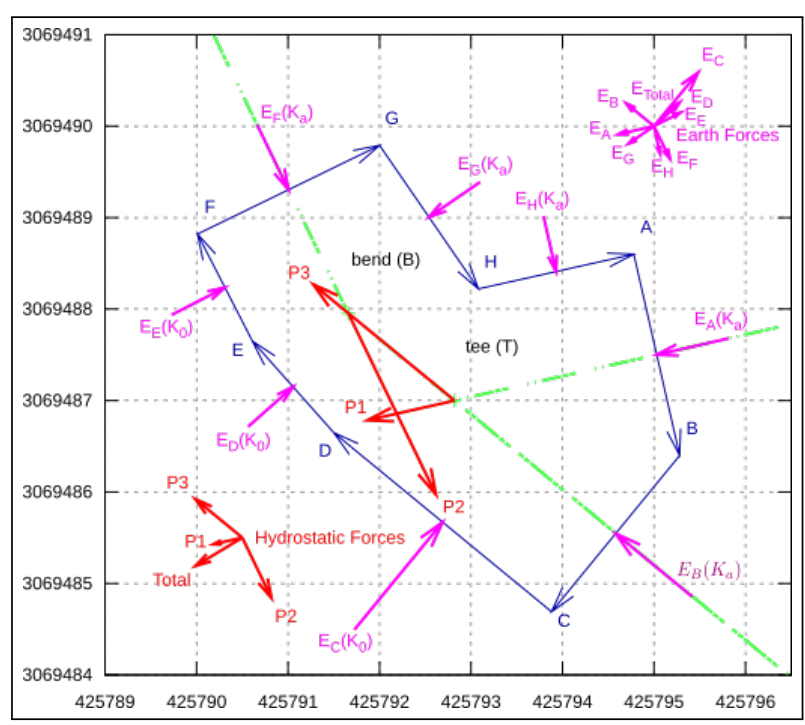

Figure 12: All Forces Acting on the Anchor Block 
Table 8: Moments Calculations

\begin{tabular}{|c|r|r|r|}
\hline Force & Toe A & Toe B & Toe C \\
\hline$H_{P 1}$ & -265.03 & -166.77 & 196.65 \\
\hline$H_{P 2}$ & 241.22 & 615.79 & 258.86 \\
\hline$H_{P 3}$ & -703.40 & -908.75 & -187.77 \\
\hline$E_{A}$ & -193.75 & -118.98 & 153.25 \\
\hline$E_{B}$ & -115.82 & -188.60 & 0.54 \\
\hline$E_{C}$ & 288.74 & 1.05 & -365.05 \\
\hline$E_{D}$ & 138.05 & 24.72 & -155.75 \\
\hline$E_{E}$ & 151.57 & 62.49 & -143.61 \\
\hline$E_{F}$ & 43.40 & 172.90 & 79.05 \\
\hline$E_{G}$ & -151.32 & -45.36 & 156.57 \\
\hline$E_{H}$ & -0.53 & 117.40 & 91.69 \\
\hline$W_{B}$ & -2329.56 & -2637.77 & -1560.91 \\
\hline$\sum M_{+v e}$ & 862.98 & 994.35 & 936.61 \\
\hline$\sum M_{-v e}$ & -3759.42 & -4066.23 & -2413.09 \\
\hline
\end{tabular}

After all the forces has been calculated final resultant is calculated by summation. The Factor of Safety against sliding can be calculated as per equation 29 or 30 . Here the final resultant is,

$R=-175.17 \hat{i}-72.51 \hat{j}-1251.12 \hat{k}$

Hence, the equations give $F o S=3.30$.

Now using the point of action of all the forces and their vector form, moments about any axis can be calculated. Dot product of that moment and the unit vector of the rotational axis(generally along a toe of the block) gives the overturning moment about that axis. The negative values mean stabilizing moments. The overturning and stabilizing moments about Toe A, B \& C are given in table 8 .

After total moments for overturning about all the toes are calculated, the Factor of Safety (FoS) of overturning for all the rotational axis are calculated. The FoS of overturning for all toes are given in table 9.

Here, minimum Factor of Safety against overturning is found to be 2.58. The safe FoS depends upon different guidelines, if it isn't satisfactory the block geometry can be changed and recalculations can be done as much as necessary.

\subsection{Discussions}

The vector formula for most forces that act on an Anchor Block has been derived by using their basic definitions were verified using the known
Table 9: Factor of Safety

\begin{tabular}{|c|r|r|r|}
\hline Toe & Overturning & Stabilizing & FoS \\
\hline A & 862.98 & 3759.42 & 4.36 \\
\hline B & 994.35 & 4066.23 & 4.09 \\
\hline C & 936.61 & 2413.09 & 2.58 \\
\hline D & 882.11 & 2461.29 & 2.79 \\
\hline E & 759.81 & 2845.00 & 3.74 \\
\hline F & 458.26 & 3812.98 & 8.32 \\
\hline G & 662.46 & 1769.70 & 2.67 \\
\hline H & 707.98 & 1902.45 & 2.69 \\
\hline
\end{tabular}

trigonometric formulae for the same forces from different books and guidelines in section 3.1.

From the section 3.2 we can see that all the forces have clear meaning in their vector form and can be easily understood in reference to the graph shown in figure 12. The direction of each force is well represented globally to check for other conditions like not having resultant's direction in the slope direction of hill, dip direction of bed rock bedding/faults, flow direction of subsurface waters, etc to look for the critical conditions. The coefficients of earth pressure can also be determined dynamically using resultant's direction, further decreasing the amount of human logic needed.

An example of intermediate steps and results of calculation was provided to demonstrate the usability of the method and formulae. It can be clearly seen that increasing the number of planes for analysis doesn't increase the effort of analysis significantly as there is no need to resolve the forces for every direction. The dot product and cross products take care of directions and components automatically.

\section{Conclusions and Recommendations}

Here, it can be seen that the trigonometric method of finding forces in 2D calculations yield the same result as the ones from Vector method. While trigonometric calculations increase in complexity when there are a lot of planes to consider, the vector method can be salable to any complex structure. Furthermore, it also supports any additional forces which might be present.

Another advantage of this method comes in analysis, with the presence of vector form of forces and their point of application, the overturning moments along any axis can be calculated instantly (a dot and a cross 
product each Vs calculation of a lot of components and their perpendicular distances to rotating axis). Unlike in traditional method where the designer would make an intellectual guess about which axis the block would be more likely to overturn and check for that axis, here we can check the Factor of Safety against all the axis without any significant additional efforts. And since the input of the whole procedure is just project parameters and alignment geometry, the calculations can be applied to different alternatives and get result with same calculation sheet(a spreadsheet) since this method has all the sign conventions dependent on the global co-ordinates without having to know the local directions. Making it even more suitable for use in automation.

There is still much space for improvements, for example the calculation of CG can be made CAD software independent (since we already have all the geometry). An iterative formula for Anchor Block geometry can be developed dependent on the alignment, pipe diameters, cover and stability parameters for design.

\section{Acknowledgments}

Partial work for this research was done as a part of Anchor Blocks design for final year project (in partial fulfilment of bachelor's degree programme in civil engineering) for Institute of Engineering titled "Pre-feasibility study on Sunkoshi Small Hydropower Project" by Gaurav Atreya, Gaurav Nepal, Gokul Dulal, Laxmi Poudel, Madhav Shrestha, and Manoj Budhathoki under the supervision of Prof. Narendra Man Shakya.

We are also grateful to TAC Hydro Consultancy Pvt. Ltd. for providing the necessary examples of anchor blocks designs in different hydropower projects. Which helped further expand the concept.

We are also grateful to Ashvin Oli and Bibek Panthi for their comments on earlier versions of the paper.

\section{Nomenclature}

* Custom defined operator between vectors which gives the vector component of the first operand in the direction of the second operand when second operand is a unit vector

$\alpha \quad$ Anticlockwise angle made by flow direction with horizontal plane(-ve for Downhill slope)
$\alpha_{L} \quad$ Coefficient of Linear Expansion

$\Delta T \quad$ Maximum difference in temperature between summer and winter

$\gamma_{s} \quad$ Unit weight of Soil

$\gamma_{s}^{\prime} \quad$ Submerged unit weight of Soil

$\gamma_{w} \quad$ Unit weight of Water

$\gamma_{p+w} \quad$ Combined Weight per length of Pipe and Water

$\hat{i} \quad$ Unit Vector along $\mathrm{X}$ axis (Easting)

$\hat{j} \quad$ Unit Vector along $\mathrm{Y}$ axis (Northing)

$\hat{k} \quad$ Unit Vector along $\mathrm{Z}$ axis (Elevation)

$\hat{n}_{i} \quad$ Unit Vector perpendicular to the area of $i_{t h}$ face towards the soil

$\hat{p} \quad$ Unit vector perpendicular to the area of sliding plane (towards the plane)

$\hat{r_{1}} \quad$ Unit Vector along the flow direction of water in the pipe

$\hat{r_{2}}$ Unit Vector which lies in the vertical place passing through $\vec{r}_{1}$ and with the direction perpendicular to $\vec{r}_{1}$

$\hat{s} \quad$ Unit vector along the sliding direction of Anchor Block

$\mu \quad$ Coefficient of friction between soil and Anchor Block base

$\mu_{s} \quad$ Coefficient of friction between saddle and pipe

$\rho_{w} \quad$ Density of Water

$\vec{E}_{i} \quad$ Force due to Earth Pressure in $i_{t h}$ face

$\vec{F}_{T} \quad$ Force due to change in temperature

$\overrightarrow{f_{\max }} \quad$ Maximum resisting friction force

$\vec{f} \quad$ Actual friction force

$\vec{H}_{b} \quad$ Hydro-static Force on bend

$\vec{H}_{b} \quad$ Hydro-static Force on reducer

$\vec{H}_{d y n} \quad$ Hydrodynamic Force in the bend

$\overrightarrow{r_{3}} \quad$ Unit Vector perpendicular to both $\overrightarrow{r_{1}}$ and $\overrightarrow{r_{2}}$ which lies in horizontal plane $(k=0)$.

$\overrightarrow{r_{p}} \quad$ Unit Vector which lies in the horizontal plane and is in the direction of pipe. 
$\overrightarrow{S_{H}} \quad$ Horizontal Seismic Force

$\overrightarrow{S_{V}} \quad$ Vertical Seismic Force

$\vec{U} \quad$ Upthrust of Ground water on Anchor Block

$\vec{W}_{1} \quad$ Weight of Pipe along $\hat{r}_{1}$

$\vec{W}_{2} \quad$ Weight of Water and Pipe along $\hat{r_{2}}$

$\vec{W}_{B} \quad$ Weight of Anchor Block

$A_{\text {base }}$ Base Area of Anchor Block projected in Horizontal Plane

$D \quad$ Internal Diameter of the pipe

E Young's modulus of Elasticity

$g \quad$ Acceleration due to gravity

$h \quad$ Piezometric Head of Ground water on Anchor Block Base

$k \quad$ Coefficient of Earth Pressure

$k_{0} \quad$ Coefficient of At-Rest Earth Pressure

$k_{a} \quad$ Coefficient of Active Earth Pressure

$k_{p} \quad$ Coefficient of Passive Earth Pressure

$L \quad$ Total distance between the Anchor block(PI) and the Nearest saddle support(center)

$l \quad$ Total length of the pipe (Sloping distance between PI)

$l_{i} \quad$ Length of $i_{t h}$ face in contact with the soil

$l_{p} \quad$ Horizontal Length of the pipe (Chainage difference between PI)

$L_{\text {pier }} \quad$ Distance between adjacent piers

$P_{i} \quad$ Force due to water pressure on $i^{\text {th }}$ force.

$Q \quad$ Discharge of water in the pipe

$t \quad$ Thickness of the Pipe

$v \quad$ velocity of water in the pipe

$K_{H} \quad$ Horizontal Seismic Coefficient

$K_{V} \quad$ Vertical Seismic Coefficient

\section{References}

Al-Gahtani, H. J. (2009). Optimum design of buried pipeline block anchors, Practice Periodical on Structural Design and Construction 14(4): 190-193.

Arora, K. (1987). Soil Mechanics and Foundation Engineering, 6 edn, A. K. Jain, Delhi.

ASCE (2012). ASCE Manuals and Reports on Engineering Practice No. 79, 2 edn, American Society of Civil Engineers.

Bhattarai, M., Adhikari, L. B., Gautam, U. P., Laurendeau, A., Labonne, C., Hoste-Colomer, R., Sèbe, O. and Hernandez, B. (2015). Overview of the large 25 April 2015 Gorkha, Nepal, Earthquake from accelerometric perspectives, Seismological Research Letters 86(6): 1540-1548.

DOED Design Guidelines: Water Conveyance System of Hydropower Projects (2007). Water Conveyance System Design Guidelines (May).

Kisan, M., Sangathan, S., Nehru, J. and Pitroda, S. G. (1984). Indian std. for design of Anchor blocks: is. 5330.1984 .

Mosonyi, E. (1991). High-Head Power Plants, Vol. 2, 3 edn, Akademiai Kaido, Budapest.

Munson, B. R., Young, D. F., Okiishi, T. H. and Huebsch, W. W. (2009). Fundamental of Fluids Mechanics, 6 edn, John Wiley \& Sons Inc.

Pandey, M. R., Tandukar, R. P., Avouac, J. P., Lavé, J. and Massot, J. P. (1995). Interseismic strain accumulation on the Himalayan crustal ramp (Nepal), Geophysical Research Letters 22(7): 751754.

Sundberg, C. (2013). Anchor block design made easy, Pipelines 2013: Pipelines and Trenchless Construction and Renewals - A Global Perspective - Proceedings of the Pipelines 2013 Conference (1): 520-533.

Varignon, P. (n.d.). Projet d'une nouvelle mécanique, paris, 1687; p, Varignon, Nouvelle Mécanique ou Statique, Paris 1725. 